\begin{tabular}{|c|c|c|c|}
\hline Eiszeitalter $u$. Gegenwart & 37 & $93-98$ & Hannover 1987 \\
\hline
\end{tabular}

\title{
Glaziäre Ablagerungen und Terrassengliederung der Weser im Raum zwischen Eisbergen und Porta Westfalica (Nordwestdeutschland)
}

\author{
Heinrich \& Agnes Wortmann *) \\ Pleistocene, stratigraphic column, terraces, moraines, fluvioglacial features, \\ periglacial features, paleosols, glacial morphology \\ North German Hills, Weser Valley, North-Rhine-Westphalia \\ TK 25: Nr. 3719, 3720, 3819, 3820
}

Im Nachlaß meines Mannes fand ich Kurzberichte, Skizzen, Profile und Tabellen, die sich auf die WeserTerrassen und Glaziärablagerungen im südlichen Portaraum beziehen und die, wie ich glaube, interessant genug sind, weitergegeben zu werden.

Eine Karte der „Morphogenese der Quartärbasis” liegt nach dem Stand von 1970 vor; sie konnte durch neuere Bohrungen ergänzt werden, wobei im engeren Portaraum und im Veltheimer Gebiet Unsicherheiten bestehen bleiben (Abb. 1).

Bezüglich der vor-elsterzeitlichen Landschaft des Portaraumes schließen wir uns der Vorstellung von DUPHORN (1974, in WOLDSTEDT \& DUPHORN) an, der bis zum Eintreffen des Elster-Eises in Nordwestdeutschland ein nur wenig eingetieftes Talsystem annimmt, das zum W hin entwässerte und dessen kommunizierendes Netz von Wasserläufen häufig nicht mit den heutigen Flußsystemen übereinstimmt.

Die neue Terrassengliederung beruht auf dem Prinzip, das WORTMANN (1968-1971) bei seinen Kartierungen nördlich des Wiehengebirges erarbeitet hat. Zur Trennung der Terrassen wurde jeweils der Zerfall des Eises zugrundegelegt. Für die Interglazialzeiten wird bei höherem Meeresspiegel und normaler Wasserführung eine etwa dem Holozän vergleichbare Flußtätigkeit angenommen.

Die neue Terrassengliederung wird in Form einer Kurztabelle vorgestellt (Tab. 1).

*) Anschrift der Autoren: Dr. habil. H. WortmanN $†$, Oberlandesgeologe a. D., Geolog. Landesamt NordrheinWestfalen. - AGNES WORTMANN, Charlottenburger Str. 19, 3400 Göttingen.
Die Bezeichnungen „Ältere” und ,Jüngere” Terrasse sind zwar umständlich, erleichtern aber die Vergleichbarkeit mit anderen Flußsystemen.

Die Unterschiede der Auffassungen von GRUPE (1930) gegenüber denen von WORTMANN bezüglich der W e s e r - Ablagerungen sind beträchtlich. Die Existenz von GRUPEs Oberterrasse im südlichen Portaraum wird abgelehnt.

Mit LỨTTIG (1954) nehmen wir an, daß der Oberterrassenlauf der Weser den Portaraum nicht erreichte, sondern aus dem Raum Hameln nach NE über Bad Münder seinen Weg in die nordwestdeutsche Tiefebene nahm. ROHDE (1983) machte allerdings wahrscheinlich, daß der OT-Lauf der Weser von Bad Münder aus durch die Deisterpforte und das Haller-Tal in das Leine-Tal (Rössing-Bemerode) verlief.

Sowohl nördlich des Gebirges (WORTMANN 19681971) als auch südlich der Weser im Lippischen Keuperbergland (SERAPHIM 1972) sind nur Drenthe-Moränen gefunden worden. Hinweise auf $\mathrm{E} l \mathrm{~s}$ te $\mathrm{r}$ $\mathrm{E}$ is b e deckung im südlichen Portaraum fehlen.

Wenn auch das Elster-Eis den südlichen Portaraum nicht berührte, so haben bei Hameln jedoch seine Ablagerungen den Weser-Lauf beeinflußt. Sie sperrten das Oberterrassental und zwangen die Weser, einen neuen Weg zu suchen. Es mehren sich die Beispiele, daß gleichzeitig mit dem Abschmelzen des ElsterEises eine Reaktivierung alter Verwerfungen, Anhebung der Achsen etc. und eine Wiederbelebung salinarer Vorgänge stattfanden, die häufig mit einer Flußverlegung verbunden waren, so auch im Raum Tündern südlich Hameln.

Nach Auffüllung der entstandenen Senke durch periglaziale Ablagerungen und Flußschotter gelang es der 
Tab. 1: Die pleistozänen Ablagerungen im südlichen Portaraum zwischen Eisbergen und Porta Westfalica (HEINRICH WoRTMANN 1976)

\begin{tabular}{|c|c|c|c|c|}
\hline \multicolumn{2}{|r|}{ Zeltabschnitte } & Eisstand & AuBerhalb der Terrassen & Weser-Terrassen \\
\hline \multicolumn{2}{|c|}{ Weichsel-Kaltzeit } & & $\begin{array}{l}\text { Jüngerer LōB, } \\
\text { periglaziale Vorgänge }\end{array}$ & $\begin{array}{l}\text { Aufschotterung der JgNT; } 2 \text {-stufig; } 4 \text { - } 8 \mathrm{~m} \text { über Talaue; keine LöBbedeckung, } \\
\text { mäBige Erosion, die Basis der ANT nicht unterschneidend; am Talrand Erhaltung der Eem-Böden }\end{array}$ \\
\hline \multicolumn{2}{|c|}{ Eem-Interglazial } & & Bodenbildung & $\begin{array}{l}\text { geringe Erosions- und Akkumulationsvorgänge; Bodenbildung (Verkittung in ÄNT), Trockenfallen der Talränder bei } \\
\text { verringerter Wasserführung }\end{array}$ \\
\hline \multirow{7}{*}{ 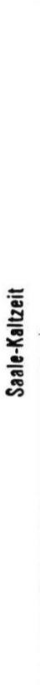 } & Warthe-Stadium & . & $\begin{array}{l}\text { LöB } \\
\text { Erniedrigung der Giazialiandschaft durch } \\
\text { périglaziale Vorgänge }\end{array}$ & $\begin{array}{l}\text { LöB } \\
\text { Aufschotterung der ÄNT } 15-18 \mathrm{~m} \text { über Talaue; nordischer Anteil } 6-10 \% \text {; Solifluktionsschichten mit stärkerem nordischen } \\
\text { Anteil; an der Basis große Drenthe-Blöcke }\end{array}$ \\
\hline & $\begin{array}{l}\text { Drenthe-Warthe- } \\
\text { Interstadial }\end{array}$ & & Tundrenzeit? & Erosions-Solifluktions-Akkumulationsvorgänge (nicht faBbar) \\
\hline & \multirow{5}{*}{ 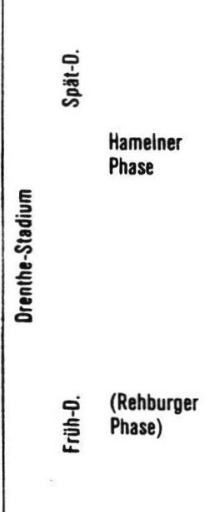 } & \multirow{3}{*}{$\begin{array}{l}\text { Zerfall des Eises } \\
\text { Weitester Eis-VorstoB } \\
\text { Eis-Halt auf dem } \\
\text { Wesergebirge }\end{array}$} & Grundmoräne auf Satzendmoräne & $\begin{array}{l}\text { starke Erosion } 5 \text { - } 8 \mathrm{~m} \text { unter Talaue } \\
\text { - Sohiental - } \\
\text { Weser durchbricht das Glaziärfluviatil; Flubverlegung nach SW durch das Vlothoer Tal zur Porta und } \\
\text { Flubveriegung nach Norden: Minden - Petershagen - Mienburg - Bremen (heutiges Weser-Tal) }\end{array}$ \\
\hline & & & \multicolumn{2}{|c|}{ Eisbedeckung } \\
\hline & & & $\begin{array}{l}\text { Autschotterung des Porta-Möllenbecker } \\
\text { Glaziärfluviatils (Satzendmoräne) } \\
\text { Staubeckentone durch Schmeizwässer vor }\end{array}$ & Abdrängung der Weser \\
\hline & & Aufbau des Eises & Periglaziale Vorgänge & $\begin{array}{l}\text { mitteistarke Erosion } 10-15 \text { m über Talaue zur JgUMT; Erosionsterrasse } \\
\text { - breites Kastental - } \\
\text { Aufschotterung der JgOMT } 25-30 \mathrm{~m} \text { über Talaue, der ÄMT auflagernd und seittich darüber hinausgehend; } \\
\text { nordischer Anteil mit } 2-4 \% \text { ziemlich regelmäBig verteilt }\end{array}$ \\
\hline & & & & mäbige Erosion; teilweise Erhaltung der Holstein-Böden \\
\hline \multicolumn{2}{|c|}{ Holstein-Interglazial } & & Bodenbildung & eingeschränkte Wasserführung; Trockenfallen der Talränder; Bodenbildung (Verkittung in ÄMT), Abnahme der Fluétïtigkeit \\
\hline \multirow{4}{*}{ 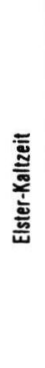 } & \multirow[t]{2}{*}{$\begin{array}{l}\text { Spāt-Elster } \\
\text { (Mindener-Phase) }\end{array}$} & & & $\begin{array}{l}\text { Aufschotterung der ÄMT } 15-20 \mathrm{~m} \text { über Talaue: nordischer Anteil } 0 \text { - 2\%; an der Basis Elster-Blöcke, } \\
\text { sehr starke Erosion } 15-20 \mathrm{~m} \text { unter Talaue; FluBverlegung: Hamein - Rintein - Veltheim - Möllbergen - Porta - Miederlande } \\
\text { - enges Rinnental - }\end{array}$ \\
\hline & & Zerfall des Eises & Periglaziale Vorgänge & $\begin{array}{l}\text { Raum Hamein } \\
\text { Auffüllung der Senke durch Weser-und Solifluktionsmaterial. } \\
\text { Reaktivierung der NS-Verwerfung Hameiner Stadtwald-Kirchohsen } \\
\text { mit Absenken des Westfliugels um } 30-40 \text { m; verstärkte Salzausiaugung. } \\
\text { Ablagerungen des Elster-Eises sperren den OT-Lauf bei Hamein ab. }\end{array}$ \\
\hline & Hoch-Elster & & (keine Eisbedeckung) & Eisbedeckung \\
\hline & Früh-Elster & & & OT-Lauf aus dem Raum Hameln nach ME \\
\hline
\end{tabular}




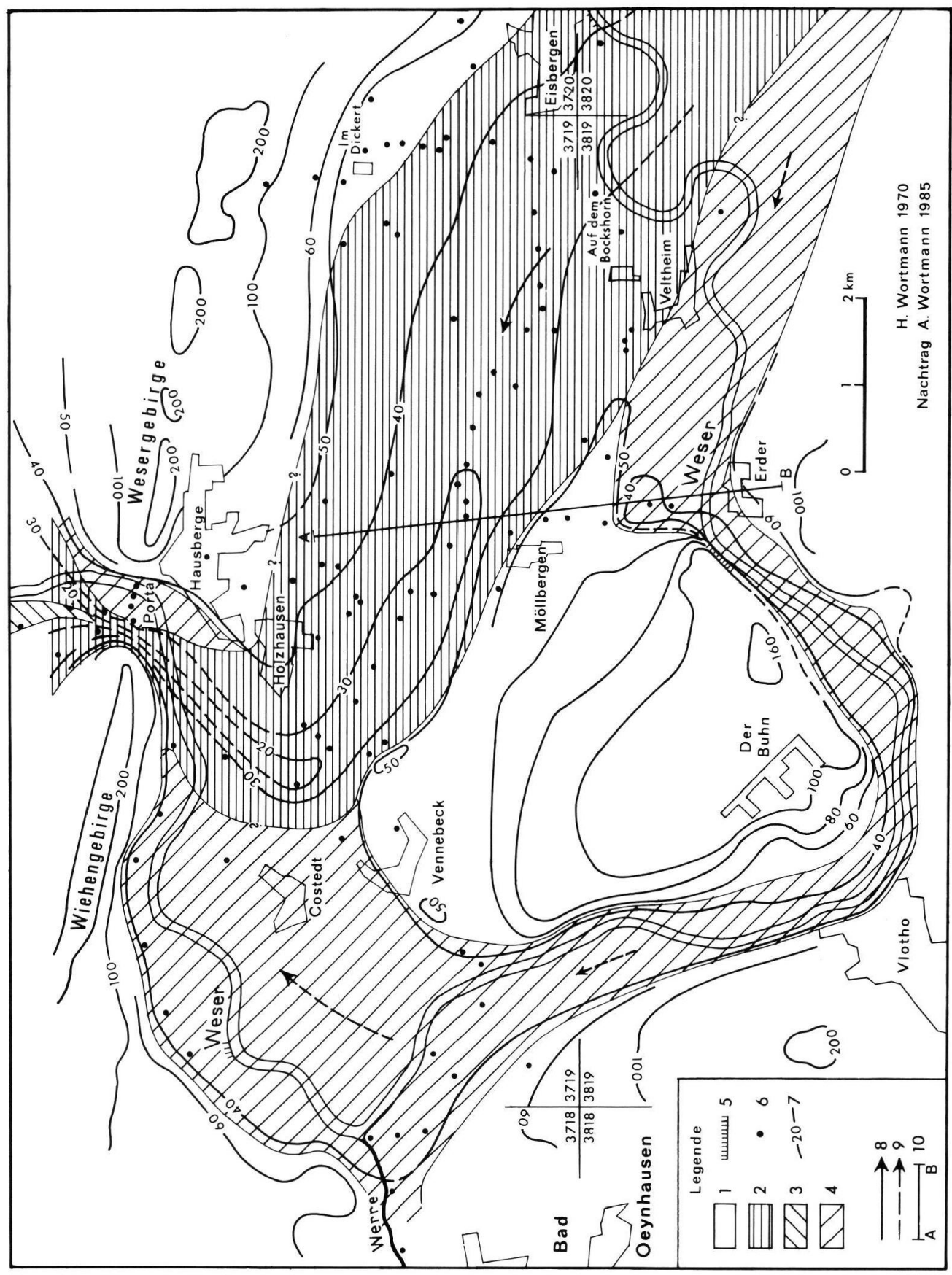

Abb. 1: Die Quartärbasis im südlichen Porta-Raum.

1 Mesozoische Gesteine

2 Fluviales Erosionsrelief aus der Zeit des Elster-Eiszerfalls bis zur Drenthe-Eisbedeckung

3 Glaziäres Rinnenrelief des enddrenthe-glazialen Eisspaltensystems in der Porta

4 Fluviales Erosionsrelief $\mathrm{n} \mathrm{a} \mathrm{c} \mathrm{h} \mathrm{dem}$ Drenthe-Eiszerfall
5 Anstehendes Mesozoikum im Weserbett

6 Bohrungen

7 Höhenlinien der Quartärbasis in NN

8 Fließrichtung der ÄMT + JgMT-Weser

9 Fließrichtung der ÄNT + JgNT-Weser

10 Lage des Profils in Abb. 2 
Weser, bei einer Tiefe von 15-20 m unter der heutigen Aue nach W auszuweichen. Sie schaffte dabei ein rinnenartiges Tal mit z. T. sehr steilen Hängen.

Westlich Rinteln durchfloß die Weser zum ersten Mal in ihrer Geschichte den südlichen Portaraum. Ihr Weg führte über Eisbergen direkt zur Porta.

Aus den Höhenwerten der Quartärbasis der bisher bekannten Bohrungen ist zu schließen, daß die Basis der sehr engen Rinne in der Porta Westfalica bei ca. $16-18 \mathrm{~m}$ üb. NN zu erwarten ist.

Die Sedimente der Aufschotterungsphase, die der Erosion folgte und im Holstein-Interglazial ihr Ende fand, werden als „Ältere Mittelterrasse” (ÄMT) bezeichnet. Ihre Mächtigkeit ist im südlichen Portaraum schwer zu bestimmen. Analog den Verhältnissen nördlich des Gebirges (WORTMANN 1971) dürfte mit $10-15 \mathrm{~m} \mathrm{zu}$ rechnen sein. Im Schotterkörper der ÄMT sind nördlich des Wiehengebirges nur maximal $0-2 \%$ nordisches Material vorhanden. Von nordischem Material freie Schichten sind relativ häufig. Zählungen im südlichen Portaraum liegen nicht vor. NAUMANN (1922) gibt größere nordische Blöcke unter der Mittelterrasse im Raum östlich Rinteln an.

Es ist anzunehmen, daß sich die Weser im HolsteinInterglazial auf ein schmaleres Bett zurückzog, so daß große Teile der ÄMT trockenfielen und einer Bodenbildung unterworfen wurden. Nördlich des Gebirges sind Reste von Bodenbildungen in Form von B- und Cca-Horizonten erhalten (WORTMANN 1971; Taf. 1). Im südlichen Portaraum sind nur gelegentlich in Bohrungen „Verkittungen” im unteren Teil der Weserschotter angegeben. Neue Untersuchungen von RÖHM (1985) bestätigen holstein-zeitliche Bodenbildungen auf elster-zeitlichen Weserablagerungen.

Die Kartierung nördlich des Wiehengebirges zeigte, daß die Erosion der Weser zu Beginn des DrentheStadials nur gering gewesen sein kann. Dafür spricht die Erhaltung der holstein-zeitlichen Böden. Es begann die Aufschotterung der $\mathrm{J}$ ü $\mathrm{n}$ g e r e n $\mathrm{M}$ i t t e l t e r rasse (Jg MT), und zwar zunächst bis zur Jüngeren O beren Mittelter r a s s e (Jg OMT). Diese bedeckte das ÄMT-Tal und ging seitlich darüber hinaus. Ihre ursprüngliche Mächtigkeit ist mit $30 \mathrm{~m}$ nicht zu gering angesetzt. Der nordische Anteil ist mit $2-4 \%$ ziemlich gleichmäßig im Schotterkörper verteilt. Schotterlagen ohne nordischen Anteil sind selten.

Beim weiteren Vorstoß des Drenthe-Eises setzte Erosion ein, die sich innerhalb der Jg OMT abspielte. Die Weser schuf ein breites Kastental mit geringem Gefälle, wobei die Erosion zur J ü n g e r e n U n t e r e $\mathrm{n}$ M i t elter rasse (Jg UMT) bei $10-15$ $\mathrm{m}$ über Talaue bei einer Höhenlage von etwa $60 \mathrm{~m}$ üb. NN abgestoppt wurde.
Die Erosion Jg OMT zur JgUMT endete, als das Drenthe-Eis die Porta blockierte Die Glaziärserie begann.

Unsere Auffassung über die Chronologie der Glaziärablagerungen ist aus dem Vergleich von WORTMANN (1975) gegenüber GRUPE (1930) und STACH (1930) in der folgenden Tabelle zu ersehen (Tab. 2).

Dank neuester Bohrungen in Barkhausen (1985), die mir Prof. SCHNEIDER (Bielefeld) freundlicherweise zur Verfügung stellte, konnte die Fortsetzung des Eisspalten-Systems am Ende des Drenthe-Stadiums (Wortmann 1968b) bis in die Porta-Enge nachgewiesen werden.

Nach GRIPP (1938) sind die glaziärfluviatilen Ablagerungen des südlichen Portaraumes eine $S$ a t $z$ e n d m or ä n e.

Während des Zerfalls des drenthe-stadialen Eises begann die Reaktivierung der Weser. Nachdem die Weser das G l a z i ä $\mathrm{r} f \mathrm{lu}$ v i a $\mathrm{t}$ i l bis Erder beseitigt hatte, mußte sie vor den Keuperhöhen des Buhnberges nach SW ins Vlothoer Tal ausweichen. Ähnlich wie beim Zerfall des Elster-Eises müssen auch in jener Zeit Reaktivierung von Verwerfungen und Salzauslaugungsvorgänge, verbunden mit Flußverlegungen, eine Rolle gespielt haben (vgl. LƯTTIG 1954). Die end-drenthestadiale Erosion der Weser reicht 5-8 $\mathrm{m}$ unter die Talaue, demnach nicht so tief wie die spät-elsterzeitliche, die $15-20 \mathrm{~m}$ erreichte. Die Talsohle scheint relativ breit und eben gewesen zu sein.

Im Drenthe/Warthe-Interstadial dürfte die Fluß-Erosion beendet gewesen sein und solifluidale Vorgänge eine Rolle gespielt haben.

Die Aufschotterung zur $\ddot{\mathrm{l}} \mathrm{lt}$ e r e n $\mathrm{N}$ i ed e r t e r r a s s e (ÄNT) erfolgte während des WartheStadiums. Die Mächtigkeit der ÄNT beträgt etwa $20 \mathrm{~m}$. Die Schotter weisen mit 6-10\% den höchsten Anteil an nordischen Geschieben auf. Häufig sind tonig-schluffige Schichten eingeschaltet; an der Basis haben sich $z$. T. sehr große nordische Blöcke aus der Drenthe-Grundmoräne angesammelt. Oft konnte ein höherer Anteil von Thüringerwald-Granit, der sonst selten vorkommt, beobachtet werden.

Wo auf dem Weg von Hameln zum Portaraum die Trennung der Älteren Niederterrasse vom Mittelterrassen-Tal erfolgte, ist noch unklar. Bei Veltheim ist die Trennung bereits vollzogen. Zur Veranschaulichung dient ein N-S-Profil, das vom Wesergebirgsrand bis zur Lippischen Pforte bei Erder reicht (Abb. 2).

Für das E e m - I n t e r g 1 a z i a 1 gilt Ähnliches wie für das Holstein-Interglazial: warmzeitliche Wasserführung in einem engeren Flußbett, gelegentliche 
Tab. 2: Die glaziären Ablagerungen im Weserraum zwischen Eisbergen und Porta Westfalica

GRUPE (1930), STACH (1930)

WORTMANN (1975)

Elster-Grundmoräne

Holstein-Warmzeit

stärkste Erosion

Saale-Kaltzeit

$\mathrm{k}$ e i n Eishalt

Bändertone auf Mittelterrasse

Saale 1 - Hauptvereisung

weiteste A usdehnung

Eisauftauen und Ablagerung

der „unteren Grundmoräne”

Eiszunge durch die Porta bis Rinteln

Bildung der $\mathrm{K}$ a mes

zwischen Eiszunge und Gebirge

„obere Grundmoräne” durch Eisoszillation

Eiszerfall, Vereinigung der beiden Grundmoränen an den Kamesrändern keine Elster-Grundmoräne

geringe Erosion

Portaverschluß durch Drentheeis, Weserstau

Eishalt

Bändertone (Beckentone) auf Jg UMT

Aufschotterung des Glaziärfluviatils zur

S a t z e ndmoräne

Keine „untere

Grundmoräne"

keine Eiszunge

Drentheeisvorstoß zur Hamelner Phase, we it este A u sdehnung

Eiszerfall, Drenthe-Grundmoräne über Satzendmoräne
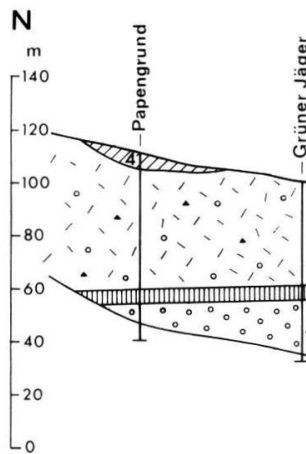

(1)
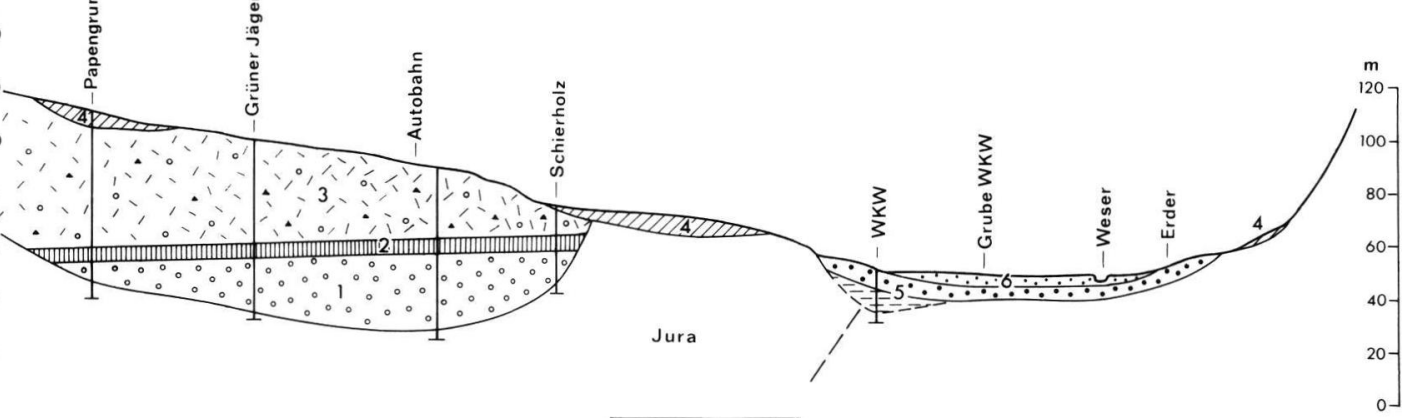

$0 \quad 500 \quad 1000 \mathrm{~m}$

Keuper

H. Wortmann 1972

Abb. 2: NS-Profil zwischen Wesergebirge und Wesertal bei Erder (Lippische Pforte).

1 Weser-Mittelterrasse, ÄMT (Spät-Elster) u. JMT (Früh-Drenthe) zusammengefaßt

2 Beckentone (schemat.)

3 Glaziärfluviatil (Satzendmoräne)
4 Drenthe-Grundmoräne

5 ÄNT (Warthe), an der Basis Ton

6 JgNT (Weichsel) 
Aufschotterung und Erosion bei Hochfluten von geringer Wirkung. Die freiliegenden ÄNT-Flächen waren Bereiche der Bodenbildung. NAUMANN (1922) erwähnt starke durchgehende Verkittungen auf randlichen „Niederterrassenschottern”. Bei Petershagen sind auf der $\ddot{A N T}$ eem-interglaziale Torfe nachgewiesen (WORTMANN 1968a: 59).

Die Erosion vor der Aufschotterung der $\mathrm{J}$ ü n g e re $\mathrm{n} \mathrm{N}$ i e d e r t e r r a s s e (Jg NT) spielte sich zu Beginn der Weichsel-Kaltzeit innerhalb des ÄNTTales ab. Sie kann nur gering gewesen sein, da die randlichen ÄNT-Schotter mit eemzeitlichen Bodenbildungen flächenhaft erhalten sind. Durchteufungen der ÄNT sind nicht bekannt.

\section{Ausblick}

Die hier zur Diskussion gestellte neue Terrassengliederung der Weser im südlichen Portaraum müßte in anderen Flußsystemen kritisch geprüft werden.

Die Aufschotterung der „Älteren Niederterrasse” fand im Warthe-Stadium statt; für die Bildungszeit der „Älteren Mittelterrasse” wird, da die Bezeichnung zum ersten Mal im Raum Minden verwendet wurde (WORTMANN 1968, 1971), der Lokalname „Mindener Phase” als jüngste Phase der ElsterKaltzeit vorgeschlagen.

\section{Schriftenverzeichnis}

Duphorn, K. et al. (1969): Der VIII. Inquakongreß in Paris 1969. - Eiszeitalter und Gegenwart 20: 252; Öhringen.

- u. a. (1973): State of reseatch on the quarternary of the Federal Republic of Germany. - Eiszeitalter und Gegenwart 23/24: 220-250; Öhringen.

- (1974): in Woldstedt, P. \& DUPHORN, K. - siehe WOLDSTEDT.
GRIPP, K. (1938): Endmoränen. - Comptes rendues de Congrès Int. de Geogr. II: 215-238; Leiden.

Grupe, O. (1930): Die Kamesbildungen des Weserberglandes. - Jb. Pr. Geol. L.-Anst., 51: 350-370; Berlin.

LƯTTIG, G. (1954): Alt- und mittelpleistozäne Eistandlagen zwischen Harz und Weser. - Geol. Jb., 70: 43-125; Hannover.

NaumanN, E. (1922): Geol. Bl. Rinteln u. Vlotho 1: 25000; Berlin.

RHode, P. (1983): Geol. Karte 1:25000 mit Erläuterungen, Bl. 3724 Pattensen. - 192 S., 43 Abb., 31 Tab., 2 Taf., 8 Kt.; Hannover.

RÖHM, H. (1985): Bau und Zusammensetzung saaleeiszeitlicher Sedimentkörper im Raum Hausberge-Veltheim/Weser. - Dipl.-Arb. Univ. Hannover: 107 S.; Hannover. - [Unveröff.].

Seraphim, E. Th. (1972): Wege und Halte des saaleeiszeitlichen Inlandeises zwischen Osning und Weser. Geol. Jb., A 3; 83 S.; Hannover.

STACH, E. (1930): Die Eisrandbildung an der Porta Westfalica. - Jb. Pr. Geol. Landesanst., 51: 174-184; Berlin.

WOLDSTEDT, P. \& DUPHORN, K. (1974): Norddeutschland und angrenzende Gebiete im Eiszeitalter. - $500 \mathrm{~S}$; Stuttgart (Köhler).

Wortmann, H. (1968a): Geol. Kt. Nordrhein-Westfalen 1: 25000 , Erl. Bl. 3619 Petershagen: 122 S.; Krefeld (Geol. L.-Amt Nordrhein Westfalen).

- (1968b): Die morphogenetische Gliederung der Quartärbasis des Wiehengebirgsvorlandes in Nordwestdeutschland. - Eiszeitalter u. Gegenwart, 19: 227-239; Öhringen.

- (1971): Geol. Kt. Nordrhein-Westfalen 1:25000, Erl. Bl. 3617 Lübbecke u. Bl. 3618 Hartum: 214 S.; Krefeld (Geol. L.-Amt Nordrhein-Westfalen). 